\title{
SOLPS simulations with electron kinetic effects
}

\author{
Menglong Zhao ${ }^{1,2}$, Alex Chankin ${ }^{1}$ and David Coster ${ }^{1}$ \\ ${ }^{1}$ Max-Planck-Institut für Plasmaphysik, Garching bei München, Boltzmannstr. 2, \\ 85748, Germany \\ ${ }^{2}$ Physik-Department E28, Technische Universität München, 85747 Garching, \\ Germany \\ E-mail: menglong.zhao@ipp.mpg.de
}

August 2018

\begin{abstract}
Power exhaust is one of the critical issues for tokamak edge plasma research. Electron kinetic effects may play an important role in future fusion devices. The KIPP code was coupled to a 1D version of SOLPS with an iterative algorithm [1,2] to study the kinetic effects in a systematic way. The KIPP-SOLPS coupling algorithm allows us to incorporate kinetic electrons into the already sophisticated fluid model (B2) self-consistently. The KIPP-SOLPS coupling simulation results with pure deuterium and with carbon impurity in 1D geometry with stagnation point upstream and target downstream are presented. These results are then compared to the results of SOLPS simulations with different flux limiters. It shows that non-local electron parallel transport contributes to the non-Maxwellian tails of electrons near the target which reduces the electron target temperature. However, the non-Maxwellian tail has negligible impacts on deuterium ionization.
\end{abstract}

\section{Introduction}

SOLPS [3] is the popular code package to simulate edge plasmas in present day tokamaks. The 2D Braginskii fluid model [4] is implemented in this code. The Braginskii model is only valid in the collisional limit where distribution functions of the plasma are close to the Maxwellian. However, due to the mobility and hence long mean free path of super-thermal electrons along magnetic field lines, electron parallel transport is typically non-local $[5,6,7]$. In the Scrape-off Layer (SOL) non-Maxwellian tails of the electron distribution function can be easily formed due to the non-local transport, especially downstream near the target where electron kinetic effects play an important role due to the fact that the electron mean free path is comparable to the electron temperature scale length [8].

Within the framework of the fluid model, earlier efforts were made both analytically and numerically to include electron kinetic effects. An analytical expression for parallel conductive heat flux formula was obtained with self-similar solutions [6, 9, 10] for the kinetic equation with a simplified collision operator. The work [11, 7, 12, 13] 
attempted to obtain a parallel heat transport equation with non-local terms by solving the simplified kinetic equation in the super-thermal limit numerically. However, these analytical formulas are not quite valid for tokamak edge plasmas where a complex geometry and strong sources are present. Some other non-local expressions were also proposed in various kinetic simulations [14, 15, 16, 17]. In refs. [18, 19, 20, 21, 22, $23,24,25,26]$ it was proposed to close fluid equations in a higher moment where a higher order macroscopic parameter $\left\langle v^{4}\right\rangle$ appeared by assuming a bi-Maxwellian distribution function [8]. However, the studies mentioned above were all carried out under the fluid model assumptions and therefore showed many limitations. Previous kinetic simulations $[27,28,29,30,31,32]$ showed that full kinetic treatment of electron parallel transport is necessary to elucidate electron kinetic effects.

The Kinetic Code for Plasma Periphery (KIPP) [33, 34, 35, 36] was developed with an aim of being coupled with SOLPS. It solves the Vlasov-Fokker-Planck equation with high accuracy of collision terms for electron parallel transport. At the same time, SOLPS is a highly sophisticated code with self-consistent recycling and physical and chemical sputtering as well as atomic physics [37]. An iterative coupling algorithm was then developed to couple KIPP with SOLPS [2], which is briefly described in section 2. The KIPP-SOLPS coupling algorithm enables one to treat electron parallel transport fully kinetically while still keeping all the physics that SOLPS has. Section 3 describes the results of simulations performed based on the coupling algorithm and comparisons of these results with those obtained with only running SOLPS.

As default, neutrals are treated kinetically with Eirene. Nevertheless, in this paper, neutrals are treated as a fluid since they are not directly involved in electron kinetic effects, and the fluid treatment of neutrals are computationally fast.

\section{Coupling kinetic electrons into SOLPS}

\subsection{Kinetic factors}

Kinetic effects mainly appear in the electron parallel transport due to high mobility of electrons along magnetic field lines. The following discussions will only be about kinetic effects in electron parallel transport. This section describes four electron related kinetic factors: electron heat conduction coefficient $c_{e}$, thermal force coefficient $k_{\|}$, sheath potential drop coefficient $\Delta \phi$ and electron sheath heat transmission coefficient

$\gamma_{e}$. Similar to definitions given in $[31,32]$, we classify the four kinetic factors into two groups: flux factors $\left(c_{e}\right.$ and $\left.k_{\|}\right)$and boundary factors $\left(\gamma_{e}\right.$ and $\left.\Delta \phi\right)$.

2.1.1. Flux factors. A 1D version of SOLPS was created for a number of applications [38, 39]. Periodic boundary conditions are implemented for the radial boundaries, forcing radial gradients to 0, to remove the radial transport (e.g. ion and electron radial particle flux densities or radial heat flux densities). Then it becomes essentially a $1 \mathrm{D}$ code in the poloidal direction. However, the transport in the poloidal 
direction in SOLPS is the sum of projections of parallel transport along the magnetic field line and the transport in the perpendicular direction (' $\perp$ ', was called diamagnetic direction in [3]) in the magnetic flux surface on the poloidal direction. The following equations are solved for electron parallel transport in this 1D version of SOLPS:

$$
\begin{gathered}
\frac{\partial n_{e}}{\partial t}+\nabla_{\|}\left(\Gamma_{e \|}\right)=S_{p}-\nabla_{\perp} \Gamma_{e \perp} \\
e n_{e} E_{\|}=R_{T_{\|}}-\nabla_{\|} p_{e} \\
\frac{\partial}{\partial t}\left(\frac{3}{2} n_{e} T_{e}\right)+\nabla_{\|}\left(\frac{3}{2} n_{e} T_{e} u_{e \|}+q_{e \|}^{\text {cond }}\right) \\
=-n_{e} T_{e} \nabla_{\|} u_{e \|}-Q_{\Delta}+S_{E e}-\nabla_{\perp} q_{e \perp}
\end{gathered}
$$

where $n_{e}, u_{e \|}, T_{e}, p_{e}$ are electron density, parallel velocity, temperature, pressure and $\Gamma_{e \|}, \Gamma_{e \perp}$ are electron parallel and perpendicular particle flux densities and $q_{e \perp}$ is electron perpendicular heat flux density. $Q_{\Delta}$ is the electron-ion energy exchange term. $E_{\|}$is the electric field along $\vec{B}$. Perpendicular particle and heat flux densities are:

$$
\begin{aligned}
& \Gamma_{e \perp}=-D_{\perp} \nabla_{\perp} n_{e} \\
& q_{e \perp}=-\chi_{e \perp} n_{e} \nabla_{\perp} T_{e}
\end{aligned}
$$

where $D_{\perp}$ and $\chi_{e \perp}$ are the anomalous diffusivity and thermal conductivity. $D_{\perp}=1.0$ and $\chi_{e \perp}=1.0$ are used in the following simulations. The second equation is the electron parallel momentum conservation equation which assumes ambipolarity of parallel plasma transport. Therefore parallel current is not considered in this work. For simplicity, later discussions are based on this assumption. The electron parallel conductive heat flux density and thermal force are given by the closure equations:

$$
\begin{aligned}
q_{e \|}^{\text {cond }} & =-c_{e} n_{e} \tau_{e} \frac{T_{e}}{m_{e}} \nabla_{\|} T_{e} \\
R_{T_{\|}} & =-k_{\|} n_{e} \nabla_{\|} T_{e}
\end{aligned}
$$

where $c_{e}=3.16, k_{\|}=0.71$ for singly charged ions. These closure equations are only valid in the collisional limit [4]. From previous kinetic simulations [40, 41, 42, 5, 35], kinetic effects appear in these two terms for arbitrary collisionality due to electron nonlocal transport $[5,6,7]$. A flux limiter is often applied to roughly capture the kinetic effects, which modifies the electron parallel heat flux density in the following way:

$$
q_{e}^{\text {cond }}=-\frac{1}{1+\frac{c_{e} \lambda_{e}}{\alpha_{e} L_{T_{e}}}} c_{e} \frac{n_{e} T_{e} \tau_{e}}{m_{e}} \nabla_{\|} T_{e}
$$

where $\lambda_{e}$ is electron mean free path and $L_{T_{e}}$ is electron temperature scale length.

2.1.2. Boundary factors. Another two kinetic factors: sheath potential drop $\Delta \phi$ and electron sheath heat transmission coefficient $\gamma_{e}$ appear in the boundary conditions for electron equations. The Debye sheath [43] is formed where the plasma interacts with the target. Since the sheath is very thin, it is usually collisionless and requires kinetic analysis. Hence the Debye sheath is not included in the SOLPS simulation domain. 
The simulation boundary is technically the sheath edge. Two boundary conditions for Eqs. (2) and (3) are required:

$$
\begin{aligned}
\phi^{t} & =\Delta \phi \frac{T_{e}}{e} \\
q_{e \|}^{t} & =\gamma_{e} T_{e} \Gamma_{t \|}
\end{aligned}
$$

where $\phi^{t}$ and $q_{e \|}^{t}$ are the potential and electron parallel flux density at the boundary. We assume that the potential at the target is 0 . In the collisional limit, these two coefficients are typically:

$$
\begin{aligned}
& \Delta \phi \approx 3 \\
& \gamma_{e}=2+\Delta \phi-1 \approx 4
\end{aligned}
$$

The presence of '-1' in Eq. 12 is due to the so-called "internal" energy conservation equation (Eq. (3)) implemented in SOLPS (details can be found in [1, 2]). However, kinetic simulations $[31,32]$ showed that electron non-local transport significantly influence these two coefficients.

\subsection{Iterative coupling algorithm}

2.2.1. KIPP. The main equation to be solved in $\operatorname{KIPP}[33,34,35]$ is the VlasovFokker-Planck equation:

$$
\frac{\partial \tilde{f}_{e}}{\partial \tilde{t}}+\tilde{v}_{\|} \nabla_{\|} \tilde{f}_{e}-\tilde{E}_{\|} \frac{\partial \tilde{f}_{e}}{\partial \tilde{v}_{\|}}=\left(\frac{\partial \tilde{f}}{\partial \tilde{t}}\right)_{\text {coll. }}+\tilde{S}_{\mathrm{E}}+\tilde{S}_{\mathrm{p}}
$$

KIPP is currently focused on electron parallel transport. " " denotes dimensionless variables, normalized by reference parameters: density $n_{0}$, temperature $T_{0}$, velocity $v_{0}$ and collision logarithm $\Lambda_{0} . T_{0}=m_{e} v_{0}^{2}$. The electron distribution function $f_{e}$ is $3 \mathrm{D}$ in phase space: two dimensions in velocity space (parallel and gyro-averaged perpendicular velocity) and one dimension in physical space along the magnetic field $\vec{B} . S_{\mathrm{E}}$ and $S_{\mathrm{p}}$ are electron energy and particle sources respectively. The collision term $\left(\frac{\partial \tilde{f}}{\partial \tilde{t}}\right)_{\text {coll. }}$ is described by the Fokker-Planck collision operator. It is linear for e-i collisions with the assumption of Maxwellian ions, but non-linear for e-e self-collisions. This code is

parallelized with MPI with one processor in charge of common jobs and others dealing with free-streaming between neighbour cells and collisions in each cell.

Eq. (13) is solved by using an operator splitting scheme [33], with parallel freestreaming for $1 / 2$ time step followed by Coulomb collision and the electric field force over one time step, followed again by the other $1 / 2$ time step free-streaming $[33,44,45]$.

2.2.2. Effective factors defined in KIPP. With KIPP, the two effective flux factors can be easily obtained based on the calculated $f_{e}$ :

$$
\begin{aligned}
c_{e f f} & =\left(\frac{1}{2} m_{e} \int f_{e} v^{2} v_{\|} d \vec{v}-\frac{5}{2} n_{e} T_{e} u_{e \|}\right) /\left(-n_{e} \tau_{e} \frac{T_{e}}{m_{e}} \nabla_{\|} T_{e}\right) \\
k_{e f f} & =\int m_{e} v^{\prime}\left(\frac{\partial f}{\partial t}\right)_{\text {coll. }} d \vec{v} /\left(-n_{e} \nabla_{\|} T_{e}\right)
\end{aligned}
$$


The calculation of the other two effective boundary factors is related to the boundary condition implemented in KIPP, which is clarified below.

The logical sheath boundary condition [46] is implemented, of which the main idea is to give a reasonable electron distribution function at the sheath edge without having to resolve the sheath region of inherently small space and time scales. A critical velocity $v_{c}\left(v_{c}>0\right)$ is determined by the assumption of the ambipolar flow through the final boundary:

$$
\Gamma_{i t \|}=\int_{v_{c}}^{\infty} f_{e} v_{\|} d \vec{v}
$$

where $\Gamma_{i t \|}$ is ion particle flux density at the boundary where the down-streaming electrons with parallel velocity larger than the critical velocity are removed from the simulation, while others are reflected back into the simulation domain and assumed to have the same velocities but in the opposite direction. With the critical velocity $v_{c}$, the two effective boundary factors can be obtained:

$$
\begin{aligned}
\Delta \phi_{e f f} & =\frac{m_{e} v_{c}^{2}}{2 T_{e t}} \\
\gamma_{e f f} & =\frac{1}{2} m_{e} \int_{v_{c}}^{\infty} f_{t} v^{2} v_{\|} d \vec{v} /\left(\Gamma_{t \|} T_{e t}\right)
\end{aligned}
$$

where $f_{t}, T_{e t}, \Gamma_{t \|}$ are electron distribution function, temperature, particle flux density at the boundary, respectively.

2.2.3. Coupling scheme. Due to very different time scales between the kinetic and fluid models, an iterative coupling algorithm was developed to couple KIPP with SOLPS [2]. SOLPS passes converged profiles of macroscopic plasma profiles $\left(n_{e}, T_{e}, T_{i}, \Gamma_{e \|}\right.$, etc.) to KIPP, while KIPP passes effective kinetic factors, calculated by Eqs. (14), (15), (17) and (18), back to SOLPS. The four kinetic factors in SOLPS are then replaced by the effective values:

$$
\begin{aligned}
c_{e} & =c_{e f f} \\
k_{\|} & =k_{e f f} \\
\Delta \phi & =\Delta \phi_{\text {eff }} \\
\gamma_{e} & =\gamma_{\text {eff }}-1
\end{aligned}
$$

This process is repeated until coupling convergence is reached. As discussed above, the '-1' difference in Eq. (22) is due to the 'internal' electron energy conservation where the coefficient ${ }^{2} \frac{3}{2}$ ' is used for the convective heat flux (details can be found in [2]). To simplify later discussions, we introduce the parameter $\gamma_{e \|}$, defined as:

$$
\gamma_{e \|}=\gamma_{e}+1
$$

Instead of $\gamma_{e}, \gamma_{e \|}$ is the electron sheath heat transmission coefficient specified for the total electron energy conservation equation, and is replaced with $\gamma_{e f f}$ in the coupling scheme. Later discussions are based on $\gamma_{e \|}$. 


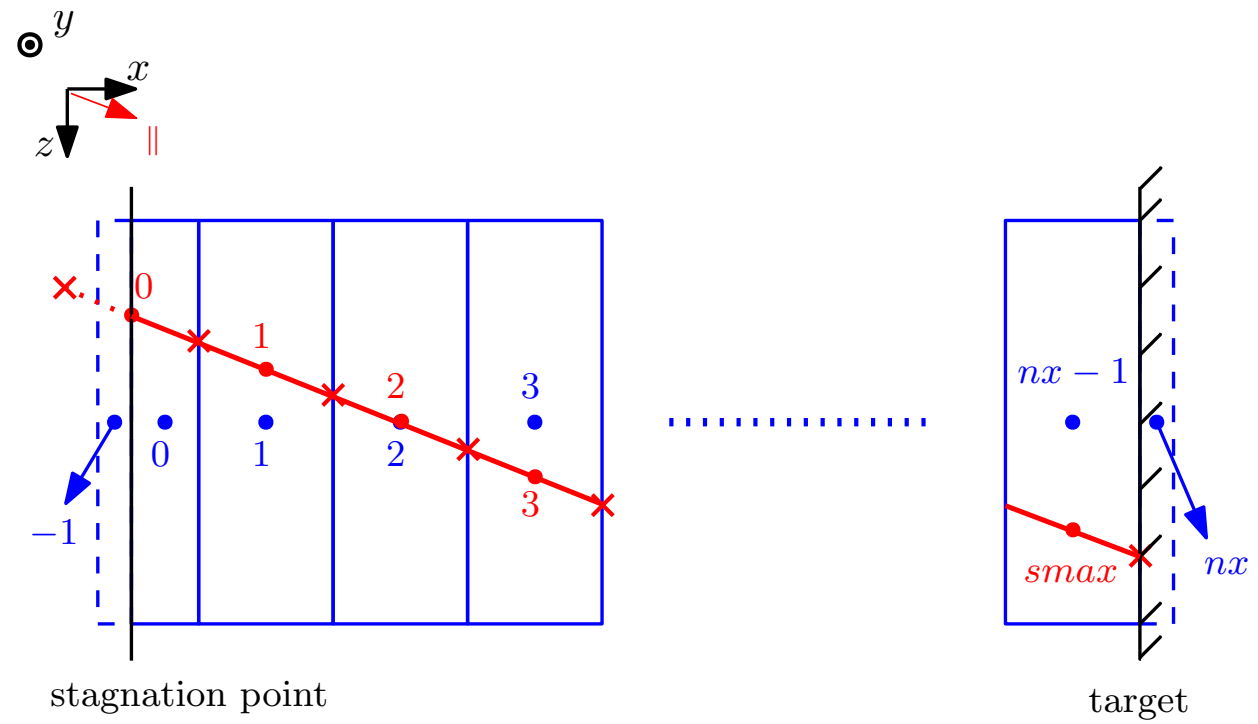

Figure 1: The grids generated in KIPP (red lines) and SOLPS (blue boxes). $x$ and $y$ are poloidal and radial directions respectively. The KIPP grid cells are generated along the direction parallel to the magnetic field with the center of cell 0 being the stagnation point and the right face of cell smax being the target, while the SOLPS grid cells are generated along the poloidal direction with two guard cells attached to the two ends. $n x=\operatorname{smax}+1$. The KIPP simulation domain is from the stagnation point (black solid line at the left end) to the target (black solid line at the right end).

\section{Simulations and discussions}

\subsection{Simulation setup}

3.1.1. The simulation geometry. The simulation geometry is taken from [2], as shown in Fig. 1, according to ASDEX-Upgrade parameters. The poloidal length is $L_{\text {pol }}=2.5 \mathrm{~m}$. The magnetic field has two components: poloidal $B_{p o l}=1.05 \times 10^{-1} \mathrm{~T}$ and toroidal $B_{\text {tor }}=9.95 \times 10^{-1} \mathrm{~T}$. The left end is the stagnation point while the right end is the target. The blue boxes are SOLPS cells and the red line is the KIPP grid generated along $\vec{B}$. The widths of cells decrease linearly from the stagnation point to the target. Cell face positions in KIPP and SOLPS are consistent with eath other. The parallel length of the KIPP simulation domain is:

$$
L_{p a r}=L_{p o l} \times \frac{\sqrt{B_{p o l}^{2}+B_{t o r}^{2}}}{B_{p o l}} \approx 25 \mathrm{~m}
$$

There are also two small guard cells attached to the two ends in SOLPS, numbered -1 and $n x$, which are created only for implementing boundary conditions.

3.1.2. Coupling setup. The work [1] showed that the iterative coupling algorithm is quite efficient to achieve coupling steady states. The density scan studies with pure deuterium plasmas were performed to justify the independence of the coupling steady state on the specific forms of coupling schemes. Carbon was added as impurity to achieve a steeper electron temperature porfile but with a medium upstream collisionality. In this 
work, the coupling simulations are performed again and then compared with SOLPS only simulations with different flux limiters and separate effective kinetic factors from the coupling steady states.

The power input is evenly distributed upstream from $x=0 \mathrm{~m}$ to $x \approx 0.83 \mathrm{~m}$. A pure deuterium plasma is used for density scan studies, and carbon is introduced as an impurity to study the case with the large temperature drop but comparatively low upstream collisionality. The recycling coefficient is at 0.999 for deuterium and 0 for carbon. The Bohm condition is assumed for ion species. For all simulations, ambipolarity of the plasma flow is assumed. The coupling scheme only allows electrons to be treated kinetically, hence ions and neutrals are still treated as fluids with default boundary conditions in SOLPS. The coupling results were shown to be independent on inital conditions [2], therefore, specifications of initial conditions for electrons are not important.

\subsection{Nomenclature}

The following nomenclature will be adopted in later discussions. For the two groups of kinetic factors, each one is given two sets of values. The values achieved in a coupling steady state are indicated as $\gamma_{e \|}^{\mathrm{ss}}, \Delta \phi^{\mathrm{ss}}, c_{e}^{\mathrm{ss}}, k_{\|}^{\mathrm{ss}}$, while those derived from the Braginskii theory are indicated as $\gamma_{e \|}^{\mathrm{c}}, \Delta \phi^{\mathrm{c}}, c_{e}^{\mathrm{c}}, k_{\|}^{\mathrm{c}}$. We will call $\gamma_{e \|}^{\mathrm{ss}}$ and $\Delta \phi^{\text {ss }}$ "kinetic boundary factors", $c_{e}^{\mathrm{ss}}$ and $k_{\|}^{\mathrm{ss}}$ "kinetic flux factors", $\gamma_{e \|}^{\mathrm{c}}$ and $\Delta \phi^{\mathrm{c}}$ "classical boundary factors", and $c_{e}^{\mathrm{c}}$ and $k_{\|}^{\mathrm{c}}$ "fluid flux factors".

Based on the coupling simulations of [2], we conclude that the so-called heat flux enhancement downstream $\left(c_{e}^{\mathrm{ss}}>c_{e}^{\mathrm{c}}\right)$ tends to flatten electron temperature rather than enhancing the heat flux. Instead of influencing the target heat flux density, the deviation of $\gamma_{e \|}^{\text {ss }}$ from $\gamma_{e \|}^{\mathrm{c}}$ causes the decrease of the target electron temperature. This indicates, counter-intuitively, that, by flattening the electron temperature profile, nonlocal transport tends to reduce the target electron temperature.

In order to explain the counter-intuitive phenomena and investigate the difference between the contributions of the two groups of kinetic factors to the modification of the plasma profiles, the kinetic boundary and flux factors are separately applied to SOLPS. Then the results are compared with the coupling results which are equivalent to those obtained by applying all four kinetic factors: $\gamma_{e \|}^{\mathrm{ss}}, \Delta \phi^{\mathrm{ss}}, c_{e}^{\mathrm{ss}}, k_{\|}^{\mathrm{ss}}$, to SOLPS.

\subsection{Density scan cases: pure deuterium}

The stagnation point density varies from $n_{u}=0.5 \times 10^{19} \mathrm{~m}^{-3}$ to $n_{u}=2.5 \times 10^{19} \mathrm{~m}^{-3}$. Alongside the full coupling cases simulated with the iterative coupling algorithm, for each stagnation point density, four SOLPS cases with and without applying kinetic boundary factors and the heat flux limiter are run:

Case A running SOLPS with fluid flux factors $\left(c_{e}=c_{e}^{\mathrm{c}}, k_{\|}=k_{\|}^{\mathrm{c}}\right)$, classical boundary factors $\left(\gamma_{e \|}=\gamma_{e \|}^{\mathrm{c}}, \Delta \phi=\Delta \phi^{\mathrm{c}}\right)$ and without heat flux limiter $\left(\alpha_{e}=\infty\right)$. 


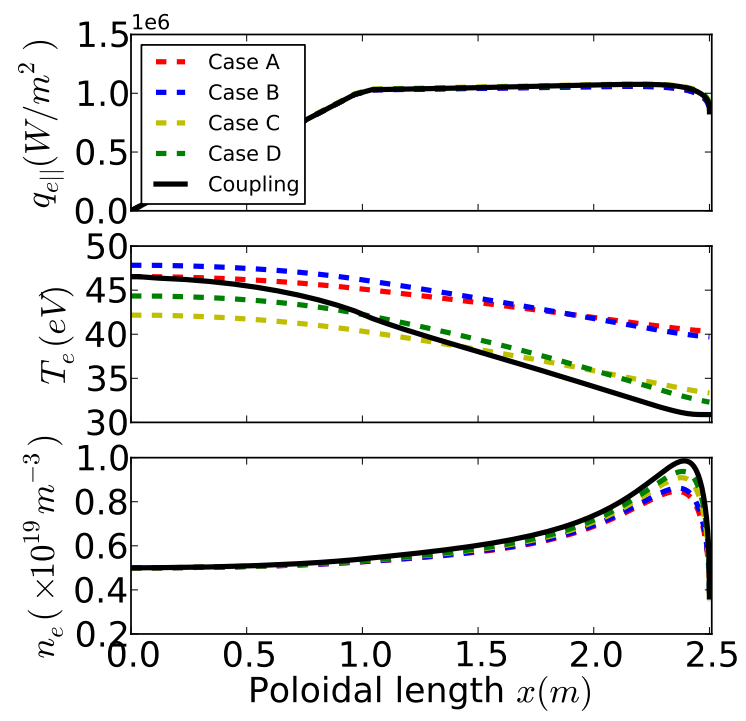

Figure 2: Profiles of $q_{e \|}, T_{e}$ and $n_{e}$ with the stagnation point density $n_{u}=0.5 \times 10^{19} \mathrm{~m}^{-3}$ are compared for the 5 cases: cases A, B, C, D and the full coupling case (labeled "Coupling"). See text for details.

Case B running SOLPS with fluid flux factors $\left(c_{e}=c_{e}^{\mathrm{c}}, k_{\|}=k_{\|}^{\mathrm{c}}\right)$, classical boundary factors $\left(\gamma_{e \|}=\gamma_{e \|}^{\mathrm{c}}, \Delta \phi=\Delta \phi^{\mathrm{c}}\right)$ and with heat flux limiter $\left(\alpha_{e}=0.3\right)$.

Case $\mathbf{C}$ running SOLPS with fluid flux factors $\left(c_{e}=c_{e}^{\mathrm{c}}, k_{\|}=k_{\|}^{\mathrm{c}}\right)$, kinetic boundary factors $\left(\gamma_{e \|}=\gamma_{e \|}^{\mathrm{ss}}, \Delta \phi=\Delta \phi^{\mathrm{ss}}\right)$ and without heat flux limiter $\left(\alpha_{e}=\infty\right)$.

Case $\mathbf{D}$ running SOLPS with fluid flux factors $\left(c_{e}=c_{e}^{\mathrm{c}}, k_{\|}=k_{\|}^{\mathrm{c}}\right)$, kinetic boundary factors $\left(\gamma_{e \|}=\gamma_{e \|}^{\mathrm{ss}}, \Delta \phi=\Delta \phi^{\mathrm{ss}}\right)$ and with heat flux limiter $\left(\alpha_{e}=0.3\right)$.

Full coupling case running SOLPS with kinetic flux factors $\left(c_{e}=c_{e}^{\mathrm{ss}}, k_{\|}=k_{\|}^{\mathrm{ss}}\right)$ and kinetic boundary factors $\left(\gamma_{e \|}=\gamma_{e \|}^{\mathrm{ss}}, \Delta \phi=\Delta \phi^{\mathrm{ss}}\right)$.

3.3.1. Low stagnation point density $n_{u}=0.5 \times 10^{19} \mathrm{~m}^{-3}$. The comparison among profiles of electron density $n_{e}$, electron temperature $T_{e}$, electron heat flux density $q_{e}$ from the 5 cases: A, B, C, D and the full coupling case with $n_{u}=0.5 \times 10^{19} \mathrm{~m}^{-3}$, is shown in Fig. 2. The upstream collisionality $\nu *$ of these cases is quite low, $\sim 6$. Here $\nu *=L / \lambda_{0}$, where $L$ is the parallel length of the simulation domain, and $\lambda_{0}$ is the electron mean free path at the stagnation point. Interestingly enough, profiles of $q_{e} \|$ from all cases are the same. Even the target electron heat flux densities $\left(q_{t \|}\right)$ are the same, although $\gamma_{e \|}$ for cases $\mathrm{A}$ and $\mathrm{B}$ is different from that for cases C, D and the full coupling case. By comparing $T_{e}$ profiles from cases $\mathrm{A}, \mathrm{C}$ and the full coupling case, it can be inferred that the deviation of $\gamma_{e \|}^{\mathrm{ss}}$ from $\gamma_{e \|}^{\mathrm{c}}$ is mainly responsible for the reduction of the target electron temperature. The heat flux limiting upstream $\left(c_{e}^{\mathrm{ss}}<c_{e}^{\mathrm{c}}\right)$ slightly increases the stagnation point temperature, while the heat flux enhancement $\left(c_{e}^{\mathrm{ss}}>c_{e}^{\mathrm{c}}\right)$ downstream flattens the electron temperature profile.

3.3.2. Medium stagnation point densities. The comparison among profiles of $n_{e}, T_{e}$ and $q_{e \|}$ for the 5 cases with $n_{u}=1.0 \times 10^{19} \mathrm{~m}^{-3}$ and $n_{u}=1.5 \times 10^{19} \mathrm{~m}^{-3}$ are shown 


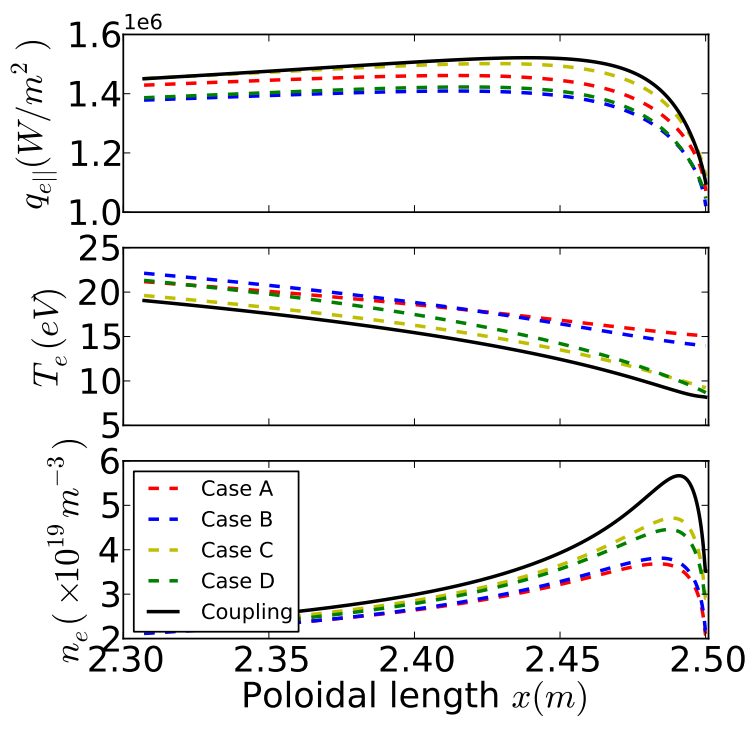

Figure 3: Profiles of $q_{e \|}, T_{e}$ and $n_{e}$ with the stagnation point density $n_{u}=1.0 \times 10^{19} \mathrm{~m}^{-3}$ are compared for the 5 cases: cases A, B, C, D and the full coupling case (labeled "Coupling"). See text for details.

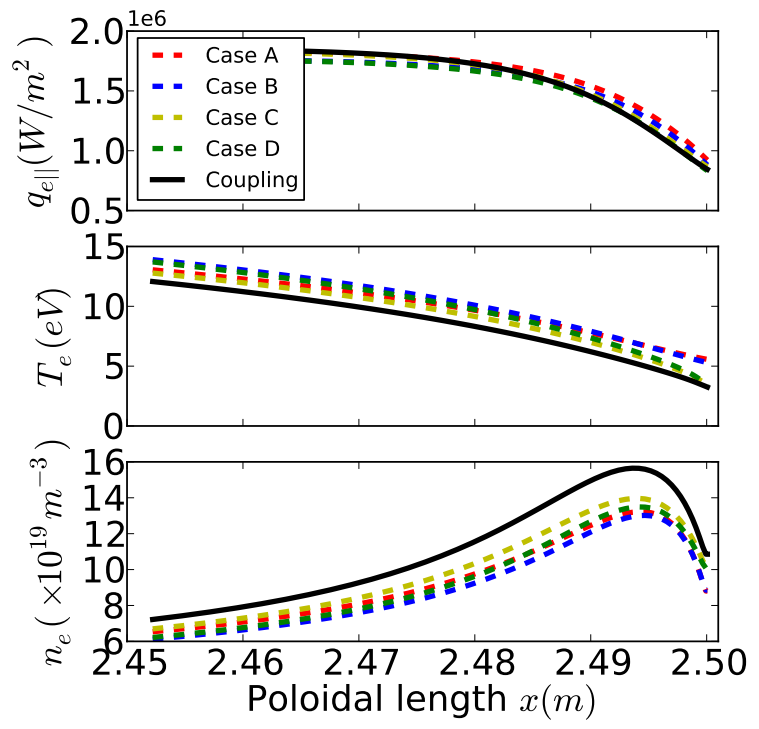

Figure 4: Profiles of $q_{e \|}, T_{e}$ and $n_{e}$ with the stagnation point density $n_{u}=1.5 \times 10^{19} \mathrm{~m}^{-3}$ are compared for the 5 cases: cases A, B, C, D and the full coupling case (labeled "Coupling"). See text for details.

in Figs. 3 (a blow-up of the region $x=2.30 \mathrm{~m}-2.50 \mathrm{~m}$ ) and 4 (a blow-up of the region $x=2.45 \mathrm{~m}-2.50 \mathrm{~m}$ ), respectively. As one can see by comparing the 5 cases for $n_{u}=1.0 \times 10^{19} \mathrm{~m}^{-3}$ and $n_{u}=1.5 \times 10^{19} \mathrm{~m}^{-3}, q_{e \|}$ is insensitive to the kinetic boundary factors and heat flux limiter, however, target electron temperatures with the kinetic boundary factors (cases C, D and the full coupling case) are almost half of those with the classical boundary factors (cases A and B). This strengthens the argument that the deviation of $\gamma_{e \|}^{\text {ss }}$ from $\gamma_{e \|}^{\mathrm{c}}$ tends to decrease the target electron temperature rather than to modify the target heat flux. From Fig. 5, where the stagnation point and target electron temperatures $\left(T_{e u}\right.$ and $\left.T_{e t}\right)$ with increasing $n_{u}$ are shown for the 5 cases, one can conclude that the deviation of $T_{e t}$ in the full coupling case from that in case $\mathrm{A}$ is mainly attributed to the deviation of $\gamma_{e \|}^{\mathrm{ss}}$ from $\gamma_{e \|}^{\mathrm{c}}$. The reason for this is investigated below.

3.3.3. Relation between $\gamma_{e \|}$ and $T_{e t}$. To explain the relation between $\gamma_{e \|}$ and $T_{e t}$, a simple analysis is given here. The target electron heat flux density scales as:

$$
q_{t \|}=\gamma_{e \|} n_{t} T_{e t} C_{s} \propto \gamma_{e \|} n_{t} T_{e t}^{3 / 2}
$$

where $n_{t} C_{s}$ is the target electron particle flux density. Upstream and downstream pressures can be related to each other by the two-point model [47]:

$$
n_{u} T_{e u} \approx 2 n_{t} T_{e t}
$$

Hence the target electron heat flux density can be transformed as:

$$
q_{t \|} \propto \gamma_{e \|} n_{u} T_{e u} T_{e t}^{1 / 2}
$$




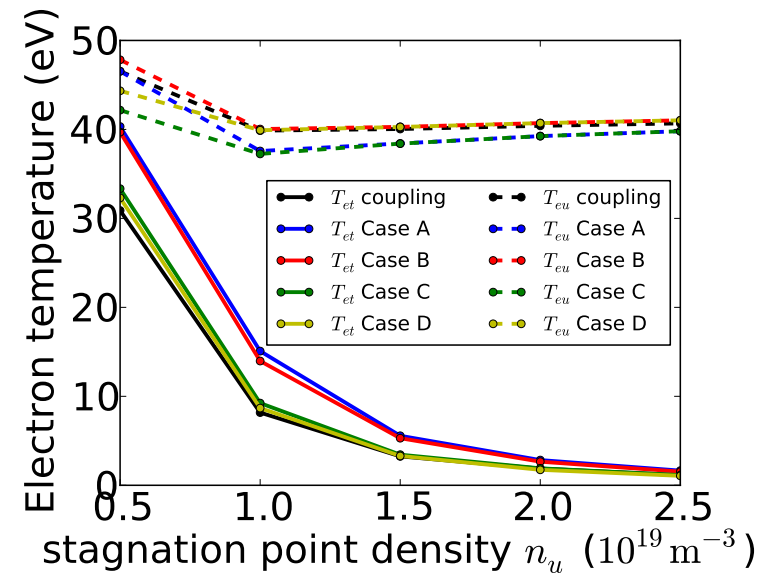

Figure 5: Comparison of the stagnation point electron temperature ( $T_{e u}$, dashed) and the target electron temperature ( $T_{e t}$, solid) among the five cases: Case A, B, C, D and the full coupling case (labeled "coupling") for various $n_{u}$.

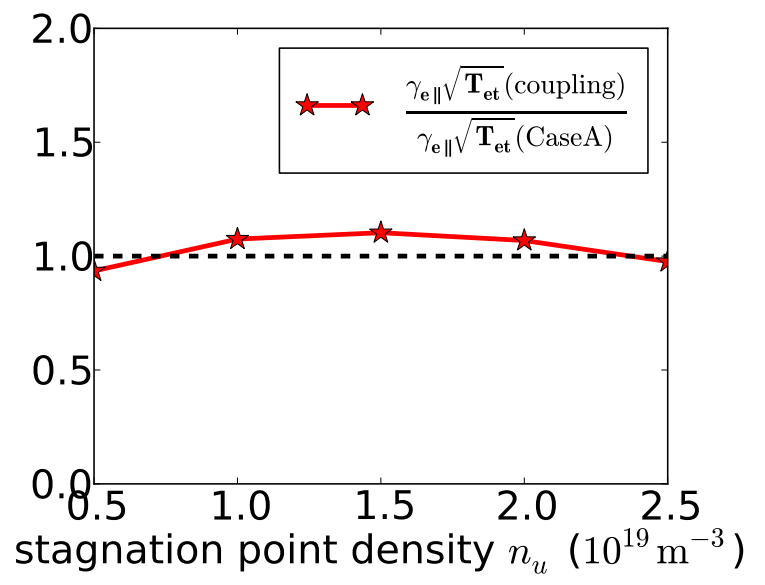

Figure 6: The ratio of $\gamma_{e \|}^{\mathrm{cpl}} \sqrt{T_{e t}^{\mathrm{cpl}}}$ to $\gamma_{e \|}^{\mathrm{A}} \sqrt{T_{e t}^{\mathrm{A}}}$ for various stagnation point densities. The horizontal black dashed line corresponds to value 1.0. This ratio is $\sim 1$, as explained in section 3.3.3.

We compare case $\mathrm{A}$ and the full coupling case at a certain $n_{u}$. As pointed out above (see Figs. 2, 3 and 4), the target electron heat flux densities for case A and the full coupling case:

$$
\begin{aligned}
& q_{t \|}^{\mathrm{A}} \propto \gamma_{e \|}^{\mathrm{A}} n_{u} T_{e u}^{\mathrm{A}} \sqrt{T_{e t}^{\mathrm{A}}} \\
& q_{t \|}^{\mathrm{cpl}} \propto \gamma_{e \|}^{\mathrm{cpl}} n_{u} T_{e u}^{\mathrm{cpl}} \sqrt{T_{e t}^{\mathrm{cpl}}}
\end{aligned}
$$

are more or less the same:

$$
q_{t \|}^{\mathrm{A}} \approx q_{t \|}^{\mathrm{cpl}}
$$

where the variables with the superscript "A" are for the Case A and "cpl" for the full coupling case. So,

$$
\begin{aligned}
& \gamma_{e \|}^{\mathrm{A}}=\gamma_{e \|}^{\mathrm{c}} \\
& \gamma_{e \|}^{\mathrm{cpl}}=\gamma_{e \|}^{\mathrm{ss}}
\end{aligned}
$$

As can be seen in Fig. 5, $T_{e u}^{\mathrm{A}} \approx T_{e u}^{\mathrm{cpl}}$, since the upstream electron temperature depends mainly on the upstream power input. Therefore:

$$
\frac{q_{t \|}^{\mathrm{A}}}{q_{t \|}^{\mathrm{cpl}}}=\frac{\gamma_{e \|}^{\mathrm{A}} \sqrt{T_{e t}^{\mathrm{A}}}}{\gamma_{e \|}^{\mathrm{cpl}} \sqrt{T_{e t}^{\mathrm{cpl}}}} \approx 1
$$

in agreement with the profile in Fig. 6. Hence

$$
\frac{T_{e t}^{\mathrm{A}}}{T_{e t}^{\mathrm{cpl}}} \approx\left(\frac{\gamma_{e \|}^{\mathrm{cpl}}}{\gamma_{e \|}^{\mathrm{A}}}\right)^{2}
$$

By comparing profiles of $n_{e}$ among the 5 cases both in Figs. 3 and 4 , one may notice that the deviation of $n_{e}$ in the full coupling case from that in case A near 


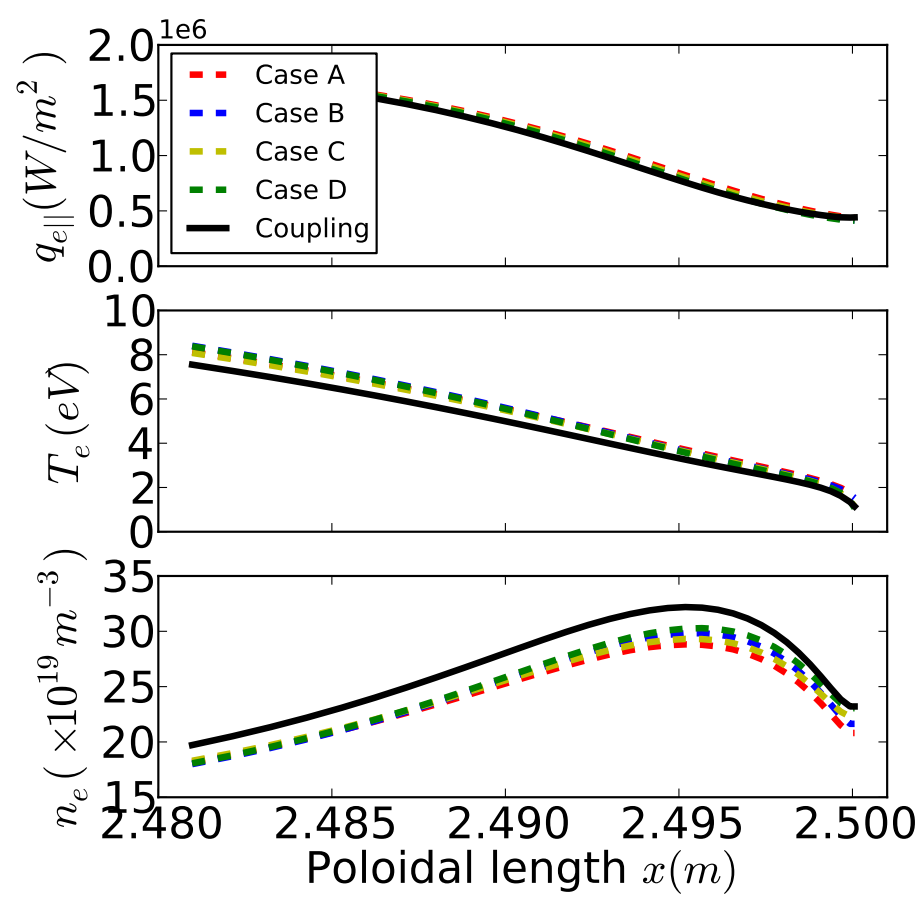

Figure 7: Profiles of $q_{e \|}, T_{e}$ and $n_{e}$ with the stagnation point density $n_{u}=2.5 \times 10^{19} \mathrm{~m}^{-3}$ are compare among the 5 cases: cases A, B, C, D and the full coupling case (details can be found in the text).

the target is mainly due to the fact that different flux factors, kinetic and fluid, are implemented differently for the two cases (case A and full coupling case). This is clarified in section 3.4.

3.3.4. High stagnation point density. Despite the difference between the target electron temperature $(\sim 1.6 \mathrm{eV})$ for cases $\mathrm{A}, \mathrm{B}$ and that $(\sim 1.15 \mathrm{eV})$ for cases $\mathrm{C}, \mathrm{D}$ and the full coupling case due to the fact explained in Eq. (34), the profiles of $n_{e}, T_{e}$ and $q_{e \|}$ in the coupling case are similar to the ones with running only SOLPS (cases A and B) as shown in Fig. 7 (a blow-up of the region $x=2.48 \mathrm{~m}-2.50 \mathrm{~m}$ ). This high collisionality case has the weakest kinetic effects in the simulation cases and further collisionalities are expected to decrease kinetic effects further.

\subsection{Activating carbon impurities}

3.4.1. Introducing $C$ in SOLPS. The future fusion device ITER [48] is planned to be run with the high confinement mode [49,50] and highly radiative divertor regime [51], where the upstream collisionality of the SOL plasma is $\nu^{*} \approx 15$, corresponding to the density scan case with $n_{u}=1.0 \times 10^{19} \mathrm{~m}^{-3}$ above. However, the coupling simulation with pure deuterium plasmas doesn't result in a large temperature drop. Carbon, used as a radiator, is introduced in the coupling simulation as an impurity [2].

Carbon impurities are activated by switching on chemical and physical impurity sputtering models in SOLPS. The constant sputtering yield for the chemical sputtering 
model is 0.095. The physical sputtering model uses TRIM database. The recycling coefficient of carbon particles (including all charged states and neutrals) is zero, while the deuterium recycling coefficient is 0.999 , the same as mentioned above. Kinetic corrections to the thermal force coefficients between electrons and carbon ions are included in the simulation.

One problem for the 1D geometry with carbon impurities, mentioned in [2], was that highly charged impurities (mainly $C^{4+}$ ) concentrated upstream due to the lack of sinks. It is suggested that the artificial carbon ion particle sink should be introduced along the simulation domain:

$$
S_{C^{i+}}=-r_{i} n_{C^{i+}} \sqrt{\frac{T_{i}}{m_{i}}} \quad i=0,6
$$

where $C^{i+}$ is the carbon impurity with charge $i$ and $r_{i}$ is the poloidal $(x)$ profile of the loss rate coefficient for $C^{i+}$ :

$$
\begin{array}{rlrl}
r_{i} & =\frac{n_{C^{i+}}(x) T_{i}(x) L_{p o l}}{\int n_{C^{i+}}(x) T_{i}(x) d x} & i & =3,6 \\
r_{i} & =0 & i & =0,2
\end{array}
$$

This sink is used for coupling and SOLPS only simulations discussed below.

3.4.2. Simulations with $C$ impurities. The deuterium ion density at the stagnation point is $n_{u}=2.2 \times 10^{19} \mathrm{~m}^{-3}$, and power input is set to match the power flux through the separatrix with constant flux density $0.132 \mathrm{MW} / \mathrm{m}^{2}$ evenly distributed in the region from $x=0 \mathrm{~m}$ to $x \approx 0.68 \mathrm{~m}$.

Similar to density scan cases, the cases A, B, C, D discussed above are run with carbon impurities and then compared with the full coupling results with carbon impurities. However, instead of applying a flux limiter $\alpha_{e}=0.3$, the case B (will be referred to as case $\mathrm{B}^{*}$ in this section in order to be distinguished from the case $\mathrm{B}$ with only deuterium in the previous section) is run with implementing the kinetic flux factors $\left(c_{e}=c_{e}^{\mathrm{ss}}\right)$ in order to investigate their effect on the $n_{e}$ profile.

Fig. 8 shows the comparison of $n_{e}, T_{e}$ and $q_{e \|}$ profiles among the five cases (only a blow-up of the region near the target $x=2.46-2.50 \mathrm{~m}$ is shown). As mentioned above, by comparing case A (or $\mathrm{B}^{*}$ ), case $\mathrm{C}$ (or D) and the full coupling case, it is clear again that the deviation of $\gamma_{e \|}^{\mathrm{ss}}$ from $\gamma_{e \|}^{\mathrm{c}}$ is mainly responsible for decreasing the target electron temperature, however, the kinetic flux factors have little impact on $T_{e t}$, as can be concluded by comparing cases A and $\mathrm{B}^{*}$.

$n_{e}$ near the target in the coupling case can be matched by running SOLPS only with the kinetic flux factors (case $\mathrm{B}^{*}$ ), as seen in Fig. 8.

Nevertheless, in difference to the results in section 3.3.2, the electron heat flux densities near and at the target are distinctly different among the five cases. It can be inferred from Fig. 9 that the discrepancy between the electron heat flux densities is mainly attributed to differences in electron energy sinks due to differences in electron temperatures and densities in this region, rather than to the influence of kinetic effects 


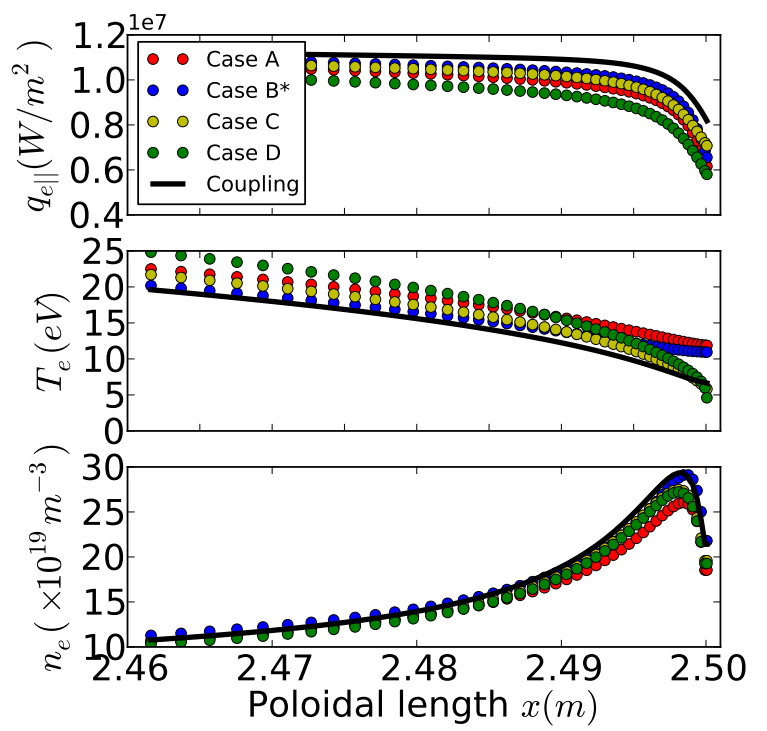

Figure 8: Profiles of $q_{e}, T_{e}$ and $n_{e}$ with the stagnation point density $n_{u}=2.2 \times 10^{19} \mathrm{~m}^{-3}$ with carbon impurity are compared among the 5 cases: cases A, B*, C, D and the full coupling case (details can be found in the text). A blowup of the region $(x=2.40 \mathrm{~m}-2.50 \mathrm{~m})$.

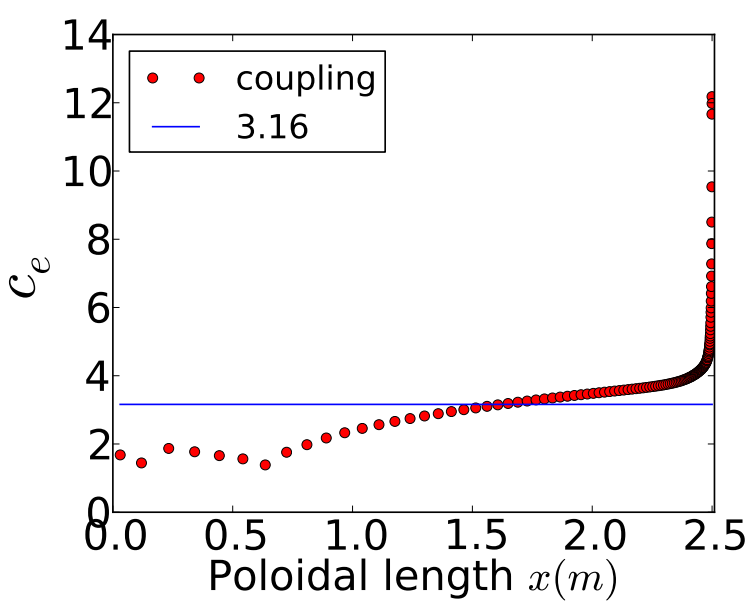

Figure 10: Profile of the electron heat conduction coefficient $\left(c_{e}=c_{e}^{\mathrm{ss}}\right)$ in the steady state full coupling case with the carbon impurity.

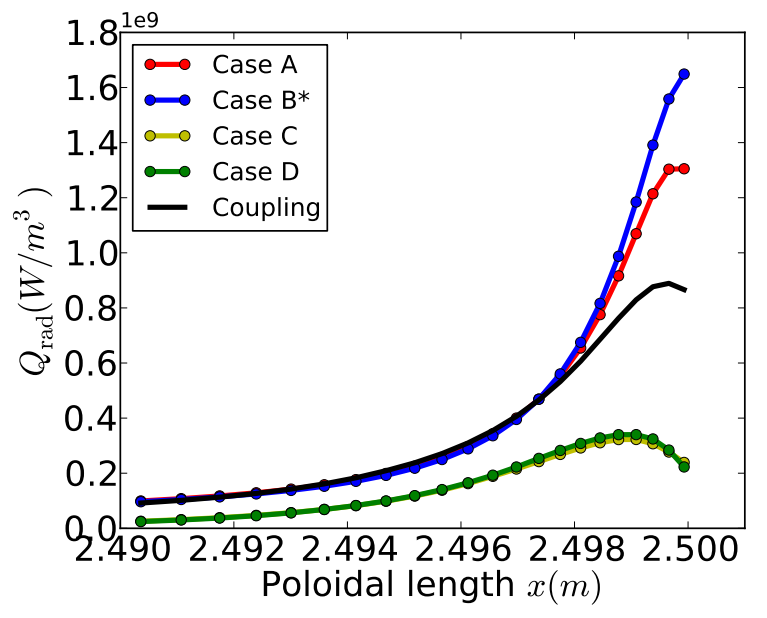

Figure 9: Profiles of the total electron radiation energy sink density (including contributions both from deuterium and carbon) in the steady state for the five cases. Only the region near the target is shown (from $x=2.49 \mathrm{~m}$ to $x=2.50 \mathrm{~m}$ ).

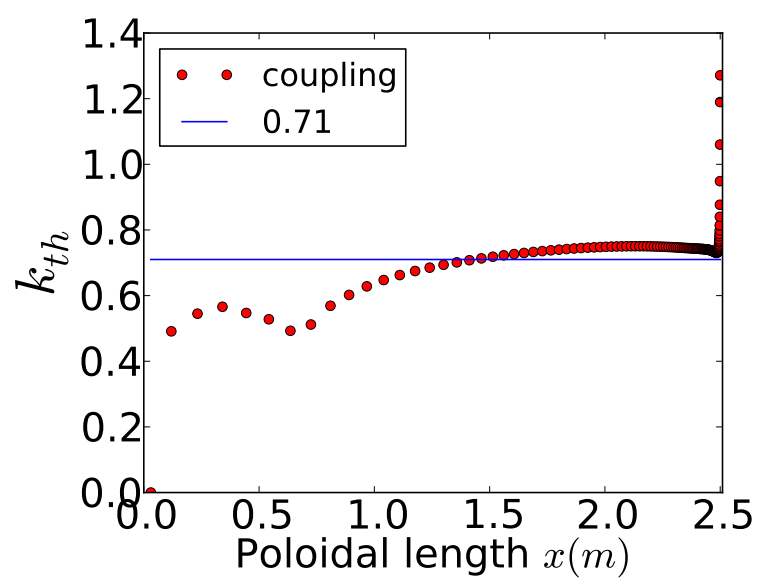

Figure 11: Profile of the thermal force coefficient $\left(k_{\|}=k_{\|}^{\text {ss }}\right)$ in the steady state full coupling case with the carbon impurity.

on the ionization rate coefficients, since kinetic atomic rates were not calculated here, which will be discussed in the next section.

Both kinetic flux and boundary factors play an important role in determining profiles of $n_{e}, T_{e}$ and therefore $q_{e \|}$. The significant deviation of $c_{e}^{\mathrm{ss}}$ and $k_{e}^{\mathrm{ss}}$ from $c_{e}^{\mathrm{c}}$ and $k_{e}^{\mathrm{c}}$ near the target (see Figs. 10 and 11), due to the extended non-Maxwellian tail and the cut-off tail $[1,27]$, tend to increase $n_{e}$, and, as discussed above, the deviation of $\gamma_{e}^{\text {ss }}$ from $\gamma_{e}^{\mathrm{c}}$ tends to decrease the target electron temperature, which together results 
in the modification of the radiation density in the near target region.

\subsection{Kinetic effects on deuterium ionization}

As pointed out in section 3.4, the non-Maxwellian effects of electron distribution functions on ionization and radiation due to non-local transport were not included in the above simulations, instead, these processes were tackled by the default subroutines in SOLPS which calculate ionization and radiation based on the ADAS database. The rate coefficients for atomic physics from ADAS, calculated based on the collision-radiative theory [52, 53] with assuming Maxwellian electrons, are tabulated and then read as an input file in SOLPS. Previous studies $[28,54,55]$ showed that the extended nonMaxwellian tail downstream near the target had substantial effects on enhancing the deuterium ionization rate coefficient. Particularly when electron temperature was lower than $3 \mathrm{eV}$, the presence of small amount (1\%) of hot electrons $(20 \mathrm{eV})$ could contribute significantly to the local ionization rate coefficient [56]. Nevertheless, the hot electron population in the previous investigations were not obtained self-consistently. With the iterative coupling algorithm, effects of non-Maxwellian tails on ionization can be selfconsistently incorporated in coupling simulations. In this work, we only investigate the influence of kinetic effects on deuterium ionization. Section 3.5.1 introduces the approach of calculating deuterium ionization rate coefficient to include non-Maxwellian effects. In section 3.5.3, the density scan cases and the case with carbon impurities are rerun with calculating deuterium ionization using this approach.

3.5.1. Collisional radiative coefficients. The collisional radiative theory gives the deuterium ionization rate coefficient $[52,53]$, as follows:

$$
S^{c r}=S_{1}-\mathbf{S}_{\mathbf{j}}\left(\mathbf{C}_{\mathbf{i j}}{ }^{-1} \mathbf{C}_{\mathbf{i} \mathbf{1}}\right)
$$

where $S_{1}$ is the rate coefficient for deuterium ionization directly from the ground state; $S_{j}$, one element in the array $\mathbf{S}_{\mathbf{j}}$, is the rate coefficient for deuterium ionization directly from the $j \operatorname{th}(j=2,3, \cdots, \operatorname{lmax})$ excited state; $C_{i j}$, one element in the matrix $\mathbf{C}_{\mathbf{i j}}$ is the coefficient for transition from the $j$ th $(j=2,3, \cdots, \operatorname{lmax})$ to $i$ th $(j=2,3, \cdots, \operatorname{lmax})$ excited states; $C_{i 1}$, one element in the array $\mathbf{C}_{\mathbf{i} 1}$, is the coefficient for transition from the ground state to $i$ th $(j=2,3, \cdots, \operatorname{lmax})$ excited state. Imax, which is referred to as 'cut-off level' in later discussions, is the highest excited state to be considered in the calculation. The parameters appeared on the right side of Eq. (38) are indicated as 'direct rate coefficients', which can be calculated by integrating $f_{e} \sigma v$ in velocity space. $\sigma$ is cross section data for a certain atomic process, which can be taken from [57, 58]. $f_{e}$ can be Maxwellian or calculated in KIPP.

3.5.2. Cut-off level specification. The rate coefficients in ADAS database were derived by resolving low excited states and regarding all higher excited states as a bundled state. A reasonable value for $\operatorname{lmax}$ can be given by comparison between the deuterium ionization coefficients calculated by Eq. (38) with Maxwellian $f_{e}$ and those from the 


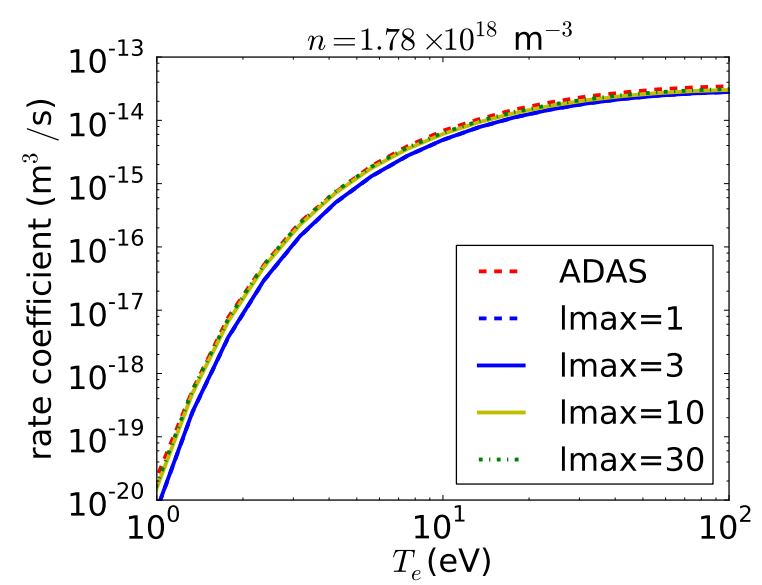

(a)

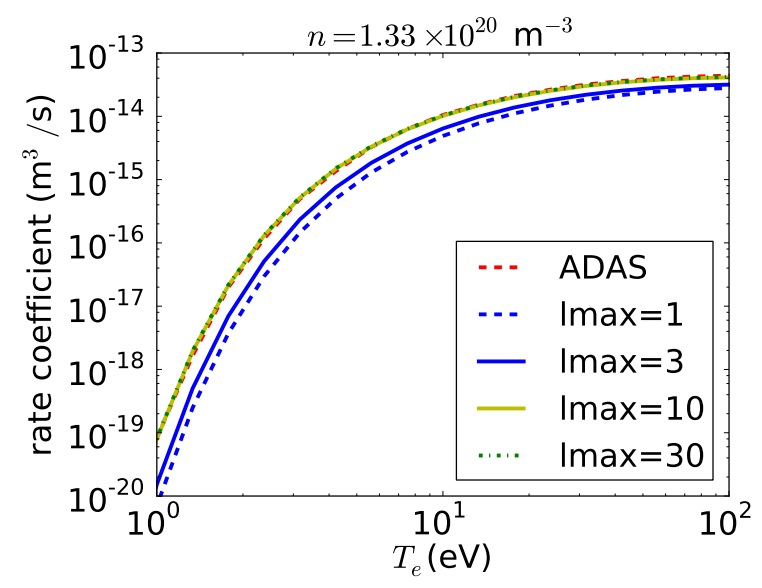

(c)

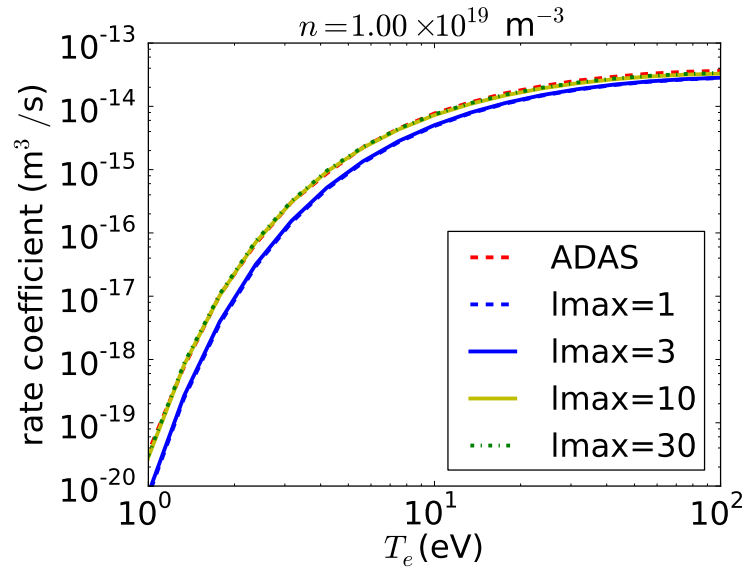

(b)

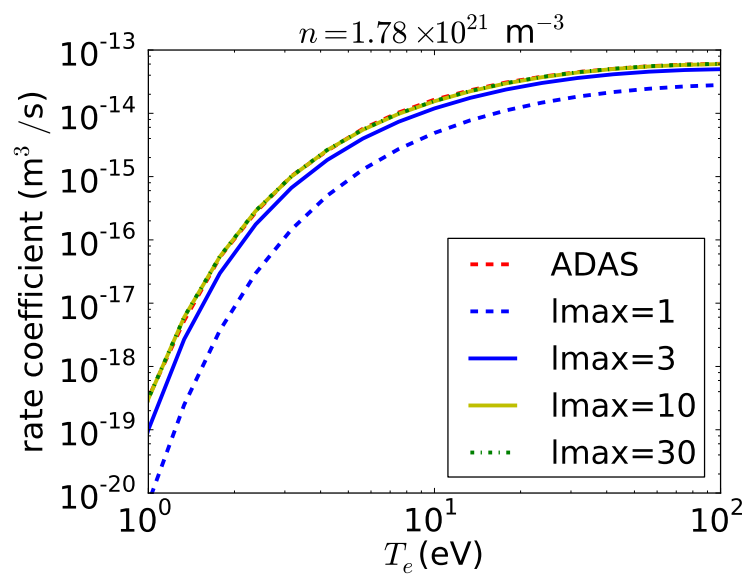

(d)

Figure 12: Deuterium ionization rate coefficients calculated from Eq. (38) with Maxwellian electrons with assuming different cut-off levels: $\operatorname{lmax}=1, \operatorname{lmax}=3, \operatorname{lmax}=10, \operatorname{lmax}=30$, compared to ADAS data for various electron densities. It seems that Eq. (38) with level $\operatorname{lmax}>10$ gives almost the same results as ADAS. When the electron density is lower than $\sim 1 \times 10^{18} \mathrm{~m}^{-3}$, the coronal assumption is valid where the ionizations are mainly from the gound state. When the electron density increases to $\sim 1 \times 10^{20} \mathrm{~m}^{-3}$, the collisional radiative process becomes important.

ADAS database, as shown in Fig. 12 for different cut-off levels $(\operatorname{lmax}=1,3,10,30)$ and various electron densities. As it can be seen, the cut-off level $\operatorname{lmax}=10$ is good enough to be chosen for the following simulations.

3.5.3. Discussions. Rather than specifying deuterium ionization rate coefficients from ADAS database, we rerun the density scan cases in section 3.3 and the case with carbon impurities in section 3.4, instead, with deuterium ionization rate coefficients calculated based on Eq. (38) in two approaches:

Approach A Calculating all involved direct rate coefficients with $f_{e}$ from KIPP:

$$
S_{i}=\int f_{e}^{\mathrm{kipp}} \sigma^{i} v d \vec{v} / n_{e}
$$




$$
C_{i j}= \begin{cases}\int f_{e}^{\mathrm{kipp}} \sigma^{i j} v d \vec{v} & \text { if } i>j \\ \int f_{e}^{\mathrm{kipp}} \sigma^{i j} v d \vec{v}+A_{i j} & \text { if } i<j \\ -\left(\sum_{k(k \neq i)} C_{k i}+n_{e} S_{i}\right) & \text { if } i=j\end{cases}
$$

where $f_{e}^{\text {kipp }}$ is the electron distribution function calculated in KIPP; $\sigma^{i}$ is cross section for deuterium ionization from the $i$ th excited state; $\sigma^{i j}$ is cross section for deuterium neutral excited state transition from the $j$ th to $i$ th states; $A_{i j}$ is Einstein coefficient; $i, j=1,2, \cdots \operatorname{lmax}$.

Approach B Calculating all involved direct rate coefficients with assuming Maxwellian distributions for electrons $f_{e}^{\mathrm{M}}$ (replacing $f_{e}$ in Eqs. (39) and (40) with $f_{e}^{\mathrm{M}}$ ).

Profiles of $n_{e}, T_{e}$ and $q_{e}$ from Approaches A and B are compared for the density scan cases. The differences between Approaches A and B are less than $0.5 \%$ for all collisionalities except the case with $n_{u}=2.5 \times 10^{19} \mathrm{~m}^{-3}$. The reason for cases with low and medium collisionalities is straightforward: the electron target temperature, which is the lowest in the $T_{e}$ profile, is not low enough so that the contribution of the non-Maxwellian tail to deuterium ionization is negligible compared to that of thermal electrons although the extended high energy tail is pronounced in the region near the target when the stagnation point density is medium $1.0-2.0 \times 10^{19} \mathrm{~m}^{-3}$.

For the high collisionality case with $n_{u}=2.5 \times 10^{19} \mathrm{~m}^{-3}$ where the target electron temperature is $\sim 1.1 \mathrm{eV}$, a significant difference between the rate coefficients from Approaches $\mathrm{A}$ and $\mathrm{B}$ at the final two cells adjacent to the target is discerned. The deuterium ionization coefficients in these two cells from Approach A are larger than those from Approach B by $\sim 10 \%$ and $\sim 50 \%$, respectively. However, the ionization rate (see Fig. ??) in these two cells $(x \approx 2.5 \mathrm{~m})$, due to low electron temperature, is several orders of magnitude lower than that in the region where ionization mostly concentrates $(x=2.48-2.49 \mathrm{~m})$ with $T_{e}=4-7 \mathrm{eV}$. The difference between rate coefficients from Approaches $\mathrm{A}$ and $\mathrm{B}$ in this region is $<0.2 \%$.

We also rerun the case with carbon impurities with calculating deuterium ionization in the two ways (A and B). The coefficients for carbon atomic physics are still taken from ADAS database. The results show that the non-Maxwellian effect on deuterium ionization has no observable impact on coupling steady state profiles although, as described in section $3.4, n_{e}, T_{e}, q_{e \|}$ and hence ionization radiation profiles have been largely influenced by electron non-local transport.

\section{Summary}

Coupling simulations successfully reproduce the expected effect of heat flux limiting $\left(c_{e}^{\mathrm{ss}}<c_{e}^{\mathrm{c}}\right)$ upstream and enhancement $\left(c_{e}^{\mathrm{ss}}>c_{e}^{\mathrm{c}}\right)$ downstream [8]. For cases with pure deuterium plasmas, based on our simulations, the heat flux limiting and enhancement factors were found to have no impact on the profile of electron heat flux, although, in the region near the target, the deviation of $c_{e}^{\text {ss }}$ from $c_{e}^{\mathrm{c}}$ is significant (e.g. by factor $3 \sim 4$ for medium upstream collisionalities). The heat flux enhancement only tends 
to flatten the electron temperature profile without influencing the absolute value much in the near target region. We conclude that the kinetic boundary factor $\gamma_{e}^{\text {ss }}$ is mainly responsible for the electron target temperature decrease compared to a fluid simulation, which leads to the change in the electron temperature profile. The SOLPS case with fluid flux factors $\left(c_{e}=c_{e}^{\mathrm{c}}, k_{\|}=k_{\|}^{\mathrm{c}}\right)$ and kinetic boundary factors $\left(\gamma_{e \|}=\gamma_{e \|}^{\mathrm{ss}}, \Delta \phi=\Delta \phi^{\mathrm{ss}}\right)$ is able to produce profiles close to those from the coupling simulation.

This, however, is not true for the case with carbon impurities. The combined effect of a remarkable decrease of the downstream electron temperature, attributed to the deviation of the kinetic boundary factors from the fluid boundary factors, and a strong density increase, caused by the difference between the kinetic flux factors and the fluid flux factors in the same region, strongly influence the ionization radiation power, thus modifying the electron heat flux profile as a result. Nevertheless, non-Maxwellian effects due to electron non-local transport on deuterium ionization and radiation can be negligible for all cases we simulated in this work. It indicates that in the conditions simulated above where the plasma profiles in density scan cases correspond to typical ASDEX-Upgrade L-mode SOL plasmas and those in the case with carbon impurities correspond to typical ASDEX-Upgrade H-mode SOL plasmas, deuterium ionization rate is not directly sensitive to the non-Maxwellian tail due to non-local transport, but can be indirectly influenced by modifications of $n_{e}$ and $T_{e}$ profiles due to the non-local transport.

We would like to conclude that the inclusion of kinetic boundary factors is always important for a fluid model to correctly predict the target electron temperature for all collisionalities we have investigated in this work. Instead of influencing the boundary heat flux, the kinetic sheath heat transmission coefficient $\gamma_{e}^{\text {ss }}$ rather modifies the target electron temperature. The increase of the upstream electron temperature due to heat flux limiting upstream is always small and hence can be neglected. The heat flux profile is determined by the energy sources and sinks rather than being directly affected by the heat flux limiting or enhancement. However, for cases with carbon impurities where the radiation concentrates downstream, the electron heat flux profile near the target is substantially modified by kinetic effects of electron parallel transport as a result of the modification of the radiation power profile due to the change of electron density and temperature in that region.

All effects investigated in this work are within the 1D geometry. Extrapolations to a 2D geometry without including kinetic effects in radial transports, which however may require gyro-kinetic codes, can be simply achieved by piling up the 1D flux tube in the radial direction. Kinetic effects on carbon ionization rates are not included in this work. However, the presence of impurities (e.g. impurity seeding) in the divertor region enhance the non-Maxwellian tail of electrons which, in turn, may have high impacts on the impurity ionization. This effect requires further studies in the future. 


\section{Acknowledgement}

This work has been carried out within the framework of the EUROfusion Consortium

and has received funding from the Euratom research and training programme 20142018 under grant agreement No 633053. The views and opinions expressed herein do not necessarily reflect those of the European Commission.

\section{References}

[1] M. Zhao, Ph.D. thesis, Technische Universität München, München, 2018, http://pubman.mpdl. mpg.de/pubman/item/escidoc:2590992/component/escidoc:2590995/IPP\%202018-10.pdf.

[2] M. Zhao, A. V. Chankin, D. P. Coster, An Iterative Algorithm of Coupling the Kinetic Code for Plasma Periphery (KIPP) with SOLPS, submitted to Computational Physics Communications.

[3] R. Schneider et al., Contrib. Plasma Phys. 46, 3 (2006).

[4] S. I. Braginskii, in Reviews of Plasma Physics (Consultants Bureau, New York, 1965), Vol. 1, Chap. Transport Processes In A Plasma, p. 205.

[5] R. Chodura, Contrib. Plasma Phys. 32, 219 (1992).

[6] S. I. Krasheninnikov, Sov. Phys. JETP 67, 2483 (1988).

[7] S. I. Krasheninnikov, Physics of Plasmas 5, 74 (1993).

[8] W. Fundamenski, Plasma Phys. Control. Fusion 47, (2005).

[9] P. J. Catto and K. Yeoh, Contrib. Plasma Phys. 38, 207 (1998).

[10] P. J. Catto and M. Grinneback, Physics Letters A 277, 323 (2000).

[11] J. R. Albritton, E. A. Williams, I. B. Bernstein, and K. P. Swartz, Phys. Rev. Lett. 57, 1887 (1986).

[12] A. M. Mirza et al., Physics Letters A 141, 56 (1989).

[13] G. Murtaza et al., Physics Letters A 144, 164 (1990).

[14] J. F. Luciani, P. Mora, and J. Virmont, Phys. Rev. Lett. 51, 18 (1983).

[15] J. R. Albritton et al., Physical Review Letters 57, 1887 (1986).

[16] B. P. Schurtz, P. D. Nicolai, and M. Busquet, Physics of Plamsas 7, 4238 (2000).

[17] J. T. Omotani and B. D. Dudson, Plasma Phys. Control. Fusion 55, 055009 (2013).

[18] E. Zawaideh, F. Najmabadi, and R. W. Conn, Physics of Fluids 29, 463 (1986).

[19] E. Zawaideh, F. Najmabadi, and R. W. Conn, Physics of Fluids 29, 3702 (1986).

[20] E. Zawaideh and N. S. Kim, Physics of Fluids 31, 3280 (1988).

[21] G. J. Radford, Contrib. Plasma Phys. 32, 297 (1992).

[22] G. J. Radford et al., Contrib. Plasma Phys. 36, 187 (1996).

[23] G. J. Radford et al., Contrib. Plasma Phys. 38, 183 (1998).

[24] Y. L. Igitkhanov and P. N. Yushmanov, Contrib. Plasma Phys. 28, 341 (1988).

[25] Y. L. Igitkhanov and A. Y. Pigarov, Journal of Nuclear Materials 176, 557 (1990).

[26] A. Bergmann et al., Contrib. Plasma Phys. 36, 192 (1996).

[27] M. Zhao, A. V. Chankin, and D. P. Coster, Nuclear Materials and Energy 12, 819 (2017).

[28] O. V. Batishchev et al., Phys. Plasmas 4, 1672 (1997).

[29] A. Froese, T. Takizuka, and M. Yagi, Contrib. Plasma Phys. 52, 534 (2012).

[30] K. Kupfer et al., Phys. Plasmas 3, 3644 (1966).

[31] D. Tskhakaya et al., Contrib. Plasma Phys. 48, 89 (2008).

[32] D. Tskhakaya et al., Journal of Nuclear Material 390, 335 (2009).

[33] A. V. Chankin, D. P. Coster, and G. Meisl, Contrib. Plasma Phys. 52, 500 (2012).

[34] A. V. Chankin and D. P. Coster, Contrib. Plasma Phys. 54, 493 (2014).

[35] A. V. Chankin and D. P. Coster, Journal of Nuclear Materials 463, 498 (2015).

[36] G. Meisl, A. V. Chankin, and D. P. Coster, Journal of Nuclear Materials 438, (2013).

[37] D. Coster et al., Physica Scripta 2006, 9 (2006). 
[38] D. P. Coster, Journal of Nuclear Materials 390, 826 (2009).

[39] D. P. Coster, Contrib. Plasma Phys. 56, 790 (2016).

[40] R. Chodura, Contrib. Plasma Phys. 28, 303 (1988).

[41] R. Chodura, Contrib. Plasma Phys. 28, 325 (1988).

[42] R. Chodura, Contrib. Plasma Phys. 30, 153 (1990).

[43] D. Bohm, The Characteristics of Electrical Discharges in Magnetic Fields (McGraw-Hill, New York, 1949).

[44] M. M. Shoucri and R. Gagn, Journal of Computational Physics 27, 315 (1978).

[45] O. V. Batishchev et al., Journal of Plasma Physics 61, 347 (1999).

[46] S. E. Parker, R. J. Procassini, C. K. Birdsall, and B. I. Cohen, Journal of Computational Physics 104, 41 (1993).

[47] P. C. Stangeby, The Plasma Boundary of Magnetic Fusion Devices (Institute of Physics, Bristol, UK, 2000).

[48] E. D. Pietro et al., Nuclear Fusion 41, 1301 (2001).

[49] F. Wagner et al., Physical Review Letters 49, 1408 (1982).

[50] F. Wagner, Plasma Physics and Controlled Fusion 49, (2007).

[51] V. A. Soukhanovskii, Plasma Physics and Controlled Fusion 59, 064005 (2017).

[52] M. Capitelli et al., in Fundamental Aspects of Plasma Chemical Physics (Springer New York, New York, 2016), Vol. 85, Chap. Collisional-Radiative Models for Atomic Hydrogen Plasmas, p. 143.

[53] H. P. Summers et al., Plasma Phys. Control. Fusion 48, 263 (2006).

[54] L. Popova et al., Contributions to Plasma Physics 44, 252 (2004).

[55] F.Allais et al., Journal of Nuclear Materials 337, .

[56] D. P. Coster, Journal of Nuclear Materials 415, (2011).

[57] R. K. Janev and J. J. Smith, Atomic and Plasma-Material Interaction Data For Fusion (IAEA, Vienna, Austria, 1993).

[58] L. C. Johnson, Astrophysical Journal 174, 227 (1972). 